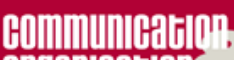
प1口.

\section{Communication et organisation}

Revue scientifique francophone en Communication organisationnelle

\section{$53 \mid 2018$}

Digitalisation et recrutement : perspectives

informationnelles et communicationnelles

\title{
Technologies numériques et travail médico-social : retour d'expérience à partir d'une recherche action formation
}

Digital technologies and work in the health care and social welfare field:

accounting for an experience from a research work on training action

\section{Audrey Bonjour et Elise Daragon}

\section{OpenEdition}

Journals

Édition électronique

URL : https://journals.openedition.org/communicationorganisation/6307

DOI : 10.4000/communicationorganisation.6307

ISSN : 1775-3546

Éditeur

Presses universitaires de Bordeaux

\section{Édition imprimée}

Date de publication : 1 juin 2018

Pagination : 197-204

ISBN : 979-10-300-0302-4

ISSN : 1168-5549

Référence électronique

Audrey Bonjour et Elise Daragon, «Technologies numériques et travail médico-social : retour d'expérience à partir d'une recherche action formation », Communication et organisation [En ligne], 53 | 2018, mis en ligne le 01 juin 2021, consulté le 28 janvier 2022. URL : http://journals.openedition.org/ communicationorganisation/6307 ; DOI : https://doi.org/10.4000/communicationorganisation.6307 


\title{
EXPÉRIENCE
}

\section{Technologies numériques et travail médico-social : retour d'expérience à partir d'une recherche action formation}

\author{
Audrey Bonjour ${ }^{1}$, Elise Daragon ${ }^{2}$
}

Résumé: Dans le cadre d'un programme de recherche, "Les technologies NUmériques au SERvice des USagers dans les établissements sociaux et médicosociaux » (NUSERUS, septembre 2015 - décembre 2018), qui a pour objectif de recenser et d'analyser les dispositifs sociotechniques utilisés dans les établissements ou services sociaux ou médico-sociaux (ESSMS), notre réflexion se focalise sur les innovations numériques et sociales qui se développent dans ces organisations. Nous mettons en œuvre une Recherche Action Formation (RAF) composée de deux phases successives, en partenariat avec le CREAI PACA et Corse (Centre interrégional d'études, d'actions et d'informations en faveur des personnes en situation de vulnérabilité Paca et Corse).

Mots-clés : Recherche Action Formation (RAF), technologies numériques (TN), établissement ou service social ou médico-social (ESSMS), travail médico-social, méthodologie participative.

Digital technologies and work in the health care and social welfare field: accounting for an experience from a research work on training action

Abstract: As part of a research program, "NUmeric Technologies in the SERvice of Users in Social and Medico-Social Institutions" (NUSERUS, September 2015 - December 2018), which aims to identify and analyze socio-technical devices used in social or medico-social institutions or services (ESSMS), our thinking focuses on the digital and social innovations that develop in these organizations. We are implementing a Research Action Training (RAF) composed of two successive phases, in partnership with the CREAI PACA and Corsica (Inter-regional Center for studies, actions and information for people in vulnerable situations Paca and Corsica).

Keywords : Research action training (RAF), digital technologies (TN), institutions or social or medico-social service (ESSMS), medico-social work, participatory methodology.

1- Audrey Bonjour est Maître de conférences en sciences de l'information et de la communication, Aix Marseille, Université de Toulon, IMSIC.

2- Elise Daragon est Maître de conférences en droit à l'université Nice Sophia Antipolis et cheurcheure au SicLab. 
Dans le cadre d'un programme de recherche, "Les technologies NUmériques au SERvice des USagers dans les établissements sociaux et médico-sociaux " (NUSERUS, septembre 2015 - décembre 2018)3 ${ }^{3}$, qui a pour objectif de recenser puis d'analyser les dispositifs sociotechniques utilisés dans les établissements ou services sociaux ou médico-sociaux (ESSMS), notre réflexion se centre sur les innovations numériques et sociales qui se développent dans ces organisations. Nous mettons en œuvre une Recherche action formation (RAF) en deux phases successives, en partenariat avec le CREAI PACA et Corse Le Centre inter-régional d'études, d'actions et d'informations en faveur des personnes en situation de vulnérabilité Paca et Corse. La première consiste, grâce à des observations participantes périphériques et des entretiens individuels et de groupes, à évaluer la place des technologies numériques - connectées ou non- dans cinq établissements de la région PACA partenaires. Nous mesurerons les conséquences de leurs utilisations sur le plan institutionnel, sur l'exercice professionnel des travailleurs sociaux et sur la prise en charge des usagers. La seconde phase prévoit la création d'une plateforme numérique de partage des pratiques relatives aux technologies numériques alimentées par les travailleurs sociaux et à destination de leurs pairs. Cette recherche sur le thème des technologies numériques dans le secteur de l'action médico-sociale vise à définir les contours de la rencontre entre ces deux mondes; la fiche de formation contractualisée s'intitule ainsi "Processus de recherche-action et accompagnement aux usages et aux pratiques éducommunicationnels ». Le concept d'éducommunication est un néologisme de forme constitué des termes " éducation " et " communication " (Soares 2000). Il ouvre vers un nouveau paradigme, c'est-à-dire une épistémologie avec de nouvelles formes et normes d'actions pour l'intervention médico-sociale (Bonjour 2014). L'éducommunication se définit comme l'ensemble des influences de la communication émanant de l'environnement d'un individu (c'està-dire des sujets mais aussi des objets), qui peut s'exercer sur lui pendant

3- Nous mobilisons plusieurs acteurs dans le projet NUSERUS : un porteur de projet institutionnel, le CREAI et deux porteurs de projet universitaire. Le projet est financé par deux Organisme Paritaires Collecteurs Agréés (OPCA) des secteurs associatif et privé. Le Groupement des établissements publics et sociaux (GEPSo) est un partenaire historique et actif du projet. Cinq établissements participent à la RAF en région PACA sur 4 départements : - quatre établissements publics que sont le Centre Hospitalier de Montfavet avec le Centre d'hébergement et de réinsertion sociale (CHRS), Avignon (84), l'établissement public St Antoine Foyer d'accueil médicalisé (FAM), Isle sur la Sorgue (84), l'Institut médicoéducatif (IME) de La Roque d'Anthéron (13), l'Établissement d'hébergement pour personnes âgées dépendantes (EHPAD) La Vençoise (06) ;

- un établissement associatif, la MAS de l'Almanarre Hyères (83). 
sa vie et qui oriente ses représentations (ses attitudes) et ses actions (son comportement) avec une attention particulière portée au lien entre les idées et les actes, et inversement.

Nous présenterons les aspects scientifiques, particulièrement le concept de recherche action formation, puis méthodologiques du projet sans pouvoir encore développer les résultats obtenus.

\section{Une recherche action formation : un dispositif participatif}

La recherche-action (RA) est une forme de recherche appliquée qui a pour but de comprendre et d'intervenir dans un certain milieu social (Tremblay et Perrier $2006: 20$ ). Notre recherche relève donc d'un dispositif de recherche-action déclinée en recherche-action-formation. Les définitions de la RA sont multiples et plusieurs typologies sont recensées (Albaladejo et Casabianca 1997 ; Gardère 2008). Elle peut se réaliser sur différents terrains avec des disciplines scientifiques diverses. Toutefois, les définitions convergent vers trois récurrences. D'abord, la RA suppose une relation d'équité entre les acteurs chercheurs et non-chercheurs qui contribuent ensemble par leur engagement et leur expertise à la concrétisation du projet, « la recherche-action ne se limite pas à une simple juxtaposition du domaine de la recherche avec celui de l'action, mais correspond, au contraire, à la relation dialogique existant entre le scientifique et le pratique " (Labbé et Courtois, 2017 : 28-29). Ensuite, la RA suppose une logique de co-construction avec les acteurs car «l'idée même de recherche-action contient cette dimension collective, aujourd'hui implicitement partagée et posée comme constitutive de toute recherche-action " (ibid.). Enfin, la pratique de la RA concilie une volonté de changement et une intention de recherche pour faire évoluer les connaissances, « ainsi, la recherche-action s'inscrit-elle dans une problématique socialement située et répond-elle à une demande sociale visant l'action et la transformation des acteurs. "Pratique sociale mixte (elle est) à l'intersection du champ scientifique, du champ de la formation, du champ éducatif » dira Perrenoud en 1995 (ibid. : 29).

La recherche action formation possède donc un lien de parenté historique avec les sciences de l'éducation qui par des formes de recherches collaboratives rapprochent recherche et théorie pour la formation des enseignants, l'enjeu étant la formation tout au long de la vie par la pratique de la recherche, cette dernière permettant aux enseignants de questionner et d'améliorer leur pédagogie. Dans une forme de continuité, 
des RAF se développent dans le champ du travail social (Fraga-Levivier et Tourrilhes 2012). À ce titre, la RAF peut s'appréhender selon trois domaines distincts mais interdépendants (Anadon et Couture 2007) : (1) Recherche, (2) Action et (3) Formation.

\section{Domaine de la recherche}

D'une part, dans la production de connaissances et de savoirs utiles pour les praticiens et la communauté scientifique, les professionnels qui sont les savants de leur réalité (Boumard 1989) « font l'expérience de la recherche » au côté des chercheurs qui « ont l'expérience de la recherche » (Cadière 2017). D'autre part, les technologies numériques sont un objet prégnant et préoccupant dans le secteur médicosocial (Meyer 2014), longtemps mises à distance dans ces établissements historiquement qualifiés de milieux clos, aujourd'hui ce sont les usagers qui apportent leur technologie ou demandent à les utiliser (Bonjour 2011).

\section{Domaine de l'action}

Le domaine de l'action se structure autour de la méthodologie de recherche et des résultats de celle-ci (lisibilité et visibilité des pratiques; ouverture d'un cours d'action). En effet, le pôle de l'action « correspond aux cycles de planification, d'action, d'observation et de réflexion sur la pratique afin de créer de nouveaux savoirs " (Anadon et Couture 2007). De plus, la recherche produit des effets en termes de lisibilité et visibilité des pratiques, c'est-à-dire rendre lisibles les pratiques des professionnels et faire valoir l'expertise et la richesse des pratiques professionnelles. Enfin, la RAF permet d'ouvrir un cours d'action (Bernard et Joule 2004) notamment à partir de l'observation participante et la participation observante en sachant que les professionnels du médico-social effectuent régulièrement des observations partagées.

\section{Domaine de la formation}

On sait que chaque professionnel est à la fois sujet et acteur et que le collectif produit de la connaissance sur la réalité de son travail. Il ne s'agit pas d'une formation académique mais d'un processus de co-construction du sens car les acquis sont produits par les acteurs dans des situations de questionnement pour faire évoluer les pratiques. En conséquence, trois enrichissements en découlent car les chercheurs, en venant à la rencontre des acteurs non-chercheurs, opèrent un triple élargissement. Ce dernier concerne, premièrement, le cadre théorique, deuxièmement 
les problématisations et troisièmement l'application à des organisations hétérogènes. En premier lieu, l'accès à une réflexion théorique passe, par exemple, par des concepts qui sont travaillés avec les professionnels tels que l'éducommunication, l'éducation critique aux médias, la médiation, la décharge cognitive, la déresponsabilisation et responsabilisation des écrits, le droit des TN, etc. En second lieu, on constate chez les professionnels une évolution, c'est-à-dire la capacité de passer des problèmes à la formulation de problématiques (observer, analyser et déduire) et enfin, on observe une projection dans l'organisation globale avec des perspectives d'institutionnalisation des pratiques. En somme, le pôle formation représente l'intégration de connaissances issues de la recherche visant à soutenir l'action avec une motivation au changement dans une visée émancipatrice.

\section{Mise en œuvre de la méthodologie}

Nous avons constitué un comité de pilotage. Son rôle est d'accompagner l'état d'avancement de la recherche en en rectifiant éventuellement les "dysfonctionnements » selon, notamment, des contraintes du secteur médico-social. De ce fait, la recherche devient un dispositif, dynamique, presque «vivant » posant l'autonomie de l'acteur comme élément primordial de l'action et de l'innovation (Peeters et Charlier, 1999). Puis, nous avons constitué des groupes de travail pluridisciplinaires dans chaque structure représentant chaque corps de métier.

Deux étapes de la phase 1 ont été identifiées lors de l'élaboration de la méthodologie : une première étape d'observation/formation de quatre jours durant laquelle des entretiens avec des professionnels et les usagers ont été menés. Nous avons réalisé des observations étudiées à partir d'une grille d'analyse des usages notamment élaborée grâce aux travaux issus du courant de la sociologie des usages; grille qui a été préalablement présentée et discutée avec les professionnels du groupe de travail de chaque établissement. Nous avons joué un rôle de catalyseur des transformations au cours de la recherche, la communication organisationnelle devient-elle ainsi organisante car la communication va être considérée comme une interaction à travers laquelle les organisations se constituent (Pascual et Bonjour, citant Taylor, Robichaud 2004).

La fin de cette étape s'est matérialisée par une restitution au groupe de travail. Lors de cet échange, l'évidence des TN nétait jamais remise en cause mais tous les paradoxes qui en découlent étaient pointés du doigt. Ainsi quatre axes de réflexion ont émergé : le partage d'information, la 
responsabilité et le positionnement éthique de l'établissement, l'impact des TN sur la relation interpersonnelle, les conséquences des TN sur les pratiques professionnelles et leur éventuelle valeur ajoutée.

La deuxième étape de la phase 1 a consisté en un accompagnement des professionnels à la production d'écrits pendant trois jours. Un fort sentiment d'appréhension entravait les professionnels mal à l'aise avec le fait de rédiger. Une démarche d'écoute et d'empathie a été nécessaire sans se départir du rôle de chercheur (Puaud 2012). L'optique de ce travail est la coproduction finale d'un ouvrage coécrit entre les chercheurs et les professionnels.

Ensuite, dans l'optique d'organiser une journée d'échange qui a eu lieu le 20 mars 2018 pour clore la phase 1 de NUSERUS en mettant en avant le travail des professionnels la co-construction d'une trame de diaporama pour la présentation lors de cette journée a été mise en œuvre. À ce jour, la phase 1 est terminée et nous allons prochainement commencer la phase 2 concernant les échanges d'utilisations novatrices des TN entre professionnels.

\section{Conclusion}

Le travail social, champ professionnel de pratiques fondé sur l'échange interpersonnel en coprésence physique n'échappe pas à l'univers d'objets connectés qui suppléent l'humain et ce, d'autant plus, qu'une injonction légale de plus en plus forte pèse sur les structures dépendantes de la réglementation et des orientations de l'action sociale4. L'histoire du travail médicosocial montre comment il est sous-tendu par nombre de paradoxes (Autès 2013; Amiguet 2004) dont les technologies numériques ne sont pas exemptes : en effet, l'opposition entre le social fondé sur la relation interpersonnelle et les technologies numériques fondées sur des algorithmes binaires contribue à faire émerger de nouveaux paradoxes.

Notre recherche action formation menée dans le cadre du projet de recherche NUSERUS montre que les technologies numériques influencent la structuration du travail social. Nos résultats nous amènent à étudier le management des technologies numériques au sein de ces organisations à travers aussi le rôle des stratégies numériques et des politiques publiques. Le projet NUSERUS fait également émerger des

4- Loi n ${ }^{\circ}$ 2015-1776 du 28/12/2015 relative à l'adaptation de la société au vieillissement, JO du 16/1/2016 et loi n 2016-1321 du 7/10/2016 pour une République du numérique, JO du 8/10/2016. 
réflexions d'ordre épistémologique qui s'insèrent dans une dynamique de science participative où les processus et les contenus peuvent être discutés et coconstruits.

\section{Bibliographie}

ALBALADEJO C., CASABIANCA F. (éds.), 1997, La recherche-action. Ambition, politique, débat, Etud. Rech. Syst. Agraire dev., 30, INRA.

AMIGUET O. \& JULIER C., 2004, Les enjeux contradictoires dans le travail social, Toulouse : ERES.

ANADON M., COUTURE C. , 2007, Présentation: La recherche participative, une préoccupation toujours vivace, in M. Anadon (dir.), La recherche participative : multiples regards (p.1-7). Saint-Nicolas: Presses de l'université du Québec.

AUTÈS M., 2013, Les paradoxes du travail social, Paris : Dunod.

BERNARD F. et VINCENT JOULE V., 2004, « Lien, sens et action : vers une communication engageante ", Communication et organisation, 24.

BONJOUR A. 2014, Pragmatisme de l'éducommunication en milieu institutionnel, p. 61-75, in Meyer, V., Les technologies numériques au service de l'usager.. Au secours du travail social ? Bordeaux : Les Études hospitalières.

BONJOUR A., 2011, Usages et pratiques socio-(ré)éducommunicationnels pour les personnes handicapées mentales. Outils informatiques et média Internet. Université Paul Verlaine-Metz.

BOUMARD P., 1989, Les savants de l'intérieur: l'analyse de la société scolaire par ses acteurs, Paris, A. Colin.

CADIERE J., 2017, « Avoir l'expérience pour la recherche Faire l'expérience de la recherche ", Forum, n' 151, (2), 3-3

FRAGA-LEVIVIER A. \& TOURRILHES C., 2012, « La recherche-action comme cadre de formation en travail social », Pensée plurielle, 30-31,(2), 243-253.

GARDERE E., 2008, « Les figures de l'acteur chercheur. Postures alternatives en débat ", p. 37-54, in Bouzon A., Meyer V., dirs., 2008, Communication des organisations, entre recherche et action, Paris, Éd. L'Harmattan.

LABBE S. et COURTOIS L. 2017, « Nécessité, bénéfices et difficultés de la coopération chercheur(s) - acteurs(s) en Recherche-Action ", La recherche en éducation, $\mathrm{n}^{\circ} 17$, p. 27-48.

PASCUAL ESPUNY C., BONJOUR A., 2016, «Processus organisants et changements de comportement dans une communication élargie aux parties prenantes : l'exemple d'une campagne d'action d'utilité publique et sociale ", in Parrini Alemano S. , 2015, Communication organisationnelle: processus communicantsprocessus organisants et leurs mediations.

PEETERS H., CHARLIER P., 1999, Contributions à une théorie du dispositif. Hermès, $n^{\circ} 25$, p. 15-23. 
Communication \& Organisation 53

PUAUD, D., 2012, L' « empathie méthodologique » en travail social, Pensée plurielle, 30-31, (2).

ROBERT R. et TREMBLAY Y., 2006, « Savoir plus : outils et méthodes de travail intellectuel », Les Éditions de la Chenelière inc.

SOARES I.O., 2000, «Educomunicação, um campo de mediações », Comunicação \& Educação, São Paulo, VII (19), p. 12-24. 